\title{
Antiglycemic Effect of Water Extractable Arabinoxylan from Wheat Aleurone and Bran
}

\author{
Lovemore Nkhata Malunga, ${ }^{1}$ Marta Izydorczyk, ${ }^{2}$ and Trust Beta ${ }^{1,3}$ \\ ${ }^{1}$ Department of Food Science, University of Manitoba, Winnipeg, MB, Canada R3T 2N2 \\ ${ }^{2}$ Canadian Grain Commission, Winnipeg, MB, Canada R3C $3 G 8$ \\ ${ }^{3}$ Richardson Centre for Functional Foods \& Nutraceuticals, University of Manitoba, Winnipeg, MB, Canada R3T 2N2 \\ Correspondence should be addressed to Trust Beta; trust.beta@umanitoba.ca
}

Received 3 February 2017; Revised 12 April 2017; Accepted 26 April 2017; Published 24 May 2017

Academic Editor: C. S. Johnston

Copyright (c) 2017 Lovemore Nkhata Malunga et al. This is an open access article distributed under the Creative Commons Attribution License, which permits unrestricted use, distribution, and reproduction in any medium, provided the original work is properly cited.

\begin{abstract}
The studies on the effects of arabinoxylan (AX) polysaccharides on postprandial glucose response have resulted in contrasting results owing to the diversity in AX structures. Four water extractable AX (WEAX) extracts obtained from wheat aleurone and bran were used to investigate (a) the effect of AX on activities of $\alpha$-amylase and $\alpha$-glucosidase, (b) influence of AX chemical composition on their inhibition potency, and (c) kinetics of enzyme inhibition. $\alpha$-Amylase activity was not significantly affected by the presence WEAX fractions regardless of type or concentration. WEAX inhibited $\alpha$-glucosidase activity only when maltose was used as a substrate but not sucrose. The IC50 values of WEAX $(4.88 \pm 0.3-10.14 \pm 0.5 \mathrm{mg} / \mathrm{mL})$ were highly correlated to ferulic acid content $(R=-0.89)$, arabinose to xylose ratio $(R=-0.67)$, and relative proportions of xylose being unsubstituted $(R=0.69)$, disubstituted $(R=-0.63)$, and monosubstituted $(R=-0.76)$. The Lineweaver-Burk plot suggested an uncompetitive enzyme inhibition mode. Thus, our results suggest that antiglycemic properties of WEAX may be derived from direct inhibition of $\alpha$-glucosidase activity.
\end{abstract}

\section{Introduction}

The prevalence of type 2 diabetes is increasing globally. Diabetes is a chronic disease epitomised by high circulating plasma glucose. Thus management of postprandial glucose is critical in prevention and treatment of type 2 diabetes patients. Human intervention studies have shown that consumption of an arabinoxylan- (AX-) rich diet attenuates postprandial blood glucose levels in healthy, impaired glucose tolerance, and diabetic subjects [1-5]. In contrast, Mohlig and coworkers [6] found no effect on glucose response when healthy human subjects were fed bread rolls supplemented with AX. Animal studies have also reported mixed results on effect of AX supplementation [7, 8]. The underlying mechanisms remain unclear but it is purported that soluble fibers increase lumen viscosity thereby delaying nutrient absorption [9]. The apparent viscosity of AX solutions is affected by the asymmetrical conformation and molecular weight of AXs as well as the polymer concentration [10]. Of these three factors, concentration of $\mathrm{AX}$ seems to influence the viscosity the most [11]. Thus the effect of AXs on blood glucose is dose-dependent. Apparent viscosity of the AX solutions is also dependent on shear stress such that higher shear results in shear thinning, the behaviour characteristic for non-Newtonian fluids [10]. Recent studies suggest that viscosity effect of AX may be offset by strong intestinal peristalsis [12].

The molecular structure of $\mathrm{AX}$ is complex and heterogeneous. AXs consist of the xylan backbone made of $(1 \rightarrow 4)$ linked $\beta$-D-xylopyranosyl (Xylp) residues with $\alpha$ L-arabinofuranosyl (Araf) residues linked to the xylan backbone at $\mathrm{C}(\mathrm{O})-2$ and $\mathrm{C}(\mathrm{O})-3$ and/or at both $\mathrm{C}(\mathrm{O})-2$ and $\mathrm{C}(\mathrm{O})$ 3 positions [10]. Xylose residues may also be substituted with glucuronic acid and/or methylglucuronic acid linkages [13]. Ferulic or coumaric acid residues are ester linked to arabinose residues at $\mathrm{C}(\mathrm{O})-5$ position $[14,15]$. The ratio of arabinose to xylose, pattern of arabinose substitution, degree of feruloylation, and molecular weight vary greatly among and within cereal grains [10]. Arabinoxylans (AX) constitute the highest proportion of dietary fiber in cereals grains $(60-70 \%)$ [16] 
TABLE 1: Chemical composition and characteristics of water extractable arabinoxylan from wheat aleurone (WA) and wheat bran (WB).

\begin{tabular}{|c|c|c|c|c|}
\hline & \multicolumn{2}{|c|}{ Wheat aleurone } & \multicolumn{2}{|c|}{ Wheat bran } \\
\hline & WA- $\mathrm{f}_{50}$ & WA- $\mathrm{f}_{75}$ & $\mathrm{WB}-\mathrm{f}_{50}$ & $\mathrm{WB}-\mathrm{f}_{75}$ \\
\hline Total carbohydrate content $(\% \mathrm{w} / \mathrm{w})$ & $76.0 \pm 0.6$ & $85.7 \pm 1.0$ & $54.6 \pm 0.5$ & $81.6 \pm 1.3$ \\
\hline Protein content $(\% \mathrm{w} / \mathrm{w})$ & $8.7 \pm 0.2$ & $8.6 \pm 0.2$ & $16.9 \pm 0.3$ & $11.3 \pm 0.3$ \\
\hline Beta glucan content $(\% \mathrm{w} / \mathrm{w})$ & 0.4 & 0.7 & 12.5 & 15.7 \\
\hline Arabinoxylan content (\% w/w) & 74.0 & 83.9 & 41.5 & 66.6 \\
\hline Arabinose to xylose ratio & 0.58 & 0.44 & 0.85 & 0.56 \\
\hline Total ferulic acid content & $26.01 \pm 0.40$ & $6.53 \pm 0.20$ & $16.78 \pm 0.35$ & $4.34 \pm 0.11$ \\
\hline Uronic acid content (\%) & $0.04 \pm 0.0$ & $0.05 \pm 0.0$ & $0.08 \pm 0.0$ & $0.10 \pm 0.0$ \\
\hline Average molecular weight (kDa) & 551.0 & 677.0 & 643.0 & 468.0 \\
\hline \multicolumn{5}{|l|}{ Patten of substitution } \\
\hline Unsub- Xylp (\%) & 61 & 70 & 45.1 & 63.8 \\
\hline Mono-Xylp at C (O)-2 (\%) & 3.2 & 0.2 & 1.4 & 0.1 \\
\hline Mono-Xylp at C (O)-3 (\%) & 16.8 & 15.9 & 23.4 & 16.4 \\
\hline Total mono-Xylp (\%) & 20.1 & 16.1 & 24.8 & 16.5 \\
\hline Di-Xylp (\%) & 19.0 & 14.0 & 30.1 & 19.8 \\
\hline
\end{tabular}

Values presented as mean \pm standard deviation $(n=3)$. Un-Xylp: unsubstituted xylose residues, mono-Xylp: monosubstituted xylose residue,di-Xylp: O2 and $\mathrm{O}-3$ disubstituted xylose residues.WA- $\mathrm{f}_{50}$ and WA- $\mathrm{f}_{75}$ : water-extractable fractions from WA obtained at 50 and $75 \%$ ammonium sulphate saturation, respectively. $\mathrm{WB}-\mathrm{f}_{50}$ and $\mathrm{WB}-\mathrm{f}_{75}$ : water-extractable from WB obtained at 50 and $75 \%$ ammonium sulphate saturation, respectively.

and their content varies with source or grain fraction. AX accounts for $1.3-2.7 \% \mathrm{w} / \mathrm{w}$ of wheat [17]. Wheat aleurone and pericarp contain 20 and $45 \%$ AX, respectively [16]. Much of the AXs in wheat are water insoluble (70-86\%) [17].

Dietary digestible carbohydrates are hydrolysed to the monomeric sugars, glucose, or fructose prior to their absorption in gastrointestinal tract [18]. Starch is digested primarily to maltose and other short chain carbohydrates by salivary and pancreatic amylase. The resultant (maltose, maltotriose, and $\alpha$-limit dextrins) and sucrose are digested to glucose or fructose by the small intestinal brush border $\alpha$-glucosidases (maltase-glucoamylase and sucrase-isomaltase) [19]. Sugar absorption in the small intestine mainly involves GLUT2, GLUT5, and SGLT1 transporters [20, 21]. Thus decrease in postprandial hyperglycaemia can be attained by limiting intestinal carbohydrate digestion or uptake. Despite the enormous differences in structure of AX, most studies report very little or no detail of composition or structure of the AX used making it difficult to compare the results on effect of AX on postprandial blood glucose [22]. Very limited data also exist on effect of purified water extractable AXs on carbohydrate digestive enzymes. Thus in this study we aimed at investigating (a) the effect of AX on activities of $\alpha$ amylase and $\alpha$-glucosidase, (b) the influence of AX chemical composition on their inhibition potency, and (c) the kinetics of enzyme inhibition.

\section{Materials and Methods}

2.1. Chemicals and Reagents. A commercial wheat aleurone (Grainwise wheat aleurone) was a gift from Cargill Limited and Horizon Milling (Wichita, Kansas, USA). It consists of $4.5,15.2,7.4$, and $2.5 \%$ lipid, protein, ash, and starch, respectively. Hard red winter wheat bran was purchased locally from Bulk Barn (Winnipeg, Manitoba, Canada). Its moisture, ash, and protein content were analysed to be 5.8, 5.3, and $11.1 \%$, respectively. Wheat unmodified starch, maltose, sucrose, acarbose, porcine pancreas $\alpha$-amylase (EC 3.2.1.1, Type VI-B), amyloglucosidase (EC 3.2.1.3) from aspergillus, and intestinal acetone powders from rat were bought from Sigma-Aldrich (Milwaukee, WI, USA). Ammonium sulphate, all acids, and organic solvents were bought from Fischer Scientific (Whitby, Ontario, Canada). Maltose, sucrose, and glucose assay kit (K-MASUG 08/13) was purchased from Megazyme International Ireland (Bray, Wicklow, Ireland). All chemicals used were of analytical or HPLC grade.

2.2. Water Extractable Arabinoxylan Preparation. The endogenous enzymes were inactivated by boiling wheat bran and wheat aleurone samples $(\sim 200 \mathrm{~g})$ in $2 \mathrm{~L}$ aqueous ethanol $(80 \%, \mathrm{v} / \mathrm{v})$ at $85^{\circ} \mathrm{C}$ under reflux for 2 hours. The supernatant was discarded and the residue was air-dried in a fume-hood overnight at room temperature. Water extractable fractions were isolated from the air-dried bran or aleurone $(150 \mathrm{~g})$ at $45^{\circ} \mathrm{C}$ according to the method described by Izydorczyk and Biliaderis [23]. The water extract was destarched using $\alpha$ amylase (1821 U/L) and deproteinized by celite and fuller's earth. The purified material was fractionated by graded ammonium sulphate (AS) precipitation and fractions were obtained at 50 and $75 \%$ AS saturation. The material collected was freeze dried after being dialyzed $(12 \mathrm{kDa}$ cut-off membrane) for 48 hours. Water extractable fractions collected from wheat aleurone were labeled (WA) followed by the concentration of AS at which they were obtained (WA-f50 and WA-f75). Similarly, the collected materials from wheat bran (WB) were designated as WB-f50 and WB-f75. The chemical and structural descriptions of WEAX fractions are presented in Table 1 .

2.3. Inhibition Assay for $\alpha$-Amylase Activity. Wheat starch (300 mg) was suspended in $15 \mathrm{~mL}$ sodium phosphate buffer ( $\mathrm{pH}$ 6.9, $0.1 \mathrm{M}$ ) containing $1 \mathrm{mM}$ calcium chloride and cooked 
at $95^{\circ} \mathrm{C}$ for 15 minutes [12]. WEAX fractions $(40 \mathrm{mg})$ were dissolved in $2 \mathrm{~mL}$ sodium phosphate buffer. Samples were diluted such that the final concentration in the reaction mixture was $0.0,0.2,0.3$, and $0.5 \%(\mathrm{w} / \mathrm{v})$. Equal volumes $(200 \mu \mathrm{L}$ ) of starch and WEAX (or control) were mixed and vortexed. Starch hydrolysis was initiated by adding $70 \mu \mathrm{L}$ of porcine pancreatic $\alpha$-amylase $(130 \mathrm{U} / \mathrm{mL})$ and $40 \mu \mathrm{L}$ of fungal amyloglucosidase $(240 \mathrm{U} / \mathrm{mL})$. The reaction was stopped after 30 minutes by heating at $95^{\circ} \mathrm{C}$ for 5 minutes. The mixture was immediately cooled on ice and centrifuged (Thermo Scientific, Sorvall Legend Micro21, Germany). The supernatants were collected and analysed for glucose using Megazyme glucose test kit. Human intervention studies have reported the effectiveness of a 0.25 to $0.70 \% \mathrm{AX}$ concentration [1-3] and hence our choice of the concentration range.

\subsection{Inhibition Assay for Rat Intestinal $\alpha$-Glucosidase Activity.} The $\alpha$-glucosidase inhibitory method by Oki et al. [24] was used with modifications. Briefly, rat intestinal acetone powder (500 mg) was mixed with $10 \mathrm{~mL}$ sodium phosphate buffer $(\mathrm{pH} 6.9,0.1 \mathrm{M})$ and sonicated in ice bath for 30 seconds (12 times) with 15 seconds break to prevent heat buildup. The mixture was later centrifuged at $10000 \mathrm{~g}$ at $4^{\circ} \mathrm{C}$ for 10 minutes. The supernatant was collected and labeled rat intestinal $\alpha$ glucosidase. WEAX samples $(40 \mathrm{mg})$ were dissolved in $2 \mathrm{~mL}$ sodium phosphate buffer $(\mathrm{pH} 6.9,0.1 \mathrm{M})$. Later, $50 \mu \mathrm{L}$ rat intestinal $\alpha$-glucosidase was mixed with $100 \mu \mathrm{L}$ sample or buffer (control) and incubated at $37^{\circ} \mathrm{C}$ for 5 minutes. Fifty $\mu \mathrm{L}$ of $20 \mathrm{mM}$ sucrose or $4 \mathrm{mM}$ maltose was added and further incubated for 60 minutes (sucrose) or 30 minutes (maltose). Final WEAX fraction concentration was $0.5,0.4,0.3,0.2$, 0.1 , and $0.0 \%(\mathrm{w} / \mathrm{v})$. The enzyme activity was halted by heating at $95^{\circ} \mathrm{C}$ for 10 minutes. After centrifugation at $10000 \mathrm{~g}$ for 10 minutes, the supernatants were collected for glucose analysis using the Megazyme GOPOD glucose test kit. Alpha glucosidase (sucrase or maltase) inhibition $\%$ was calculated as $\left(1-\left[\left(A_{\text {sample }}-A_{\text {blank }}\right) /\left(A_{\text {control }}-A_{\text {blank }}\right)\right]\right) * 100$. IC50 value was determined from the plot of $\% \alpha$-glucosidase inhibition against sample concentration. Inhibition of rat intestinal $\alpha$ glucosidase with acarbose (a known $\alpha$-glucosidase inhibitor) was also done for comparison purpose. Acarbose concentrations of $1.625,3.25,4.9,6.5,9.8$, and $13 \mu \mathrm{g} / \mathrm{mL}$ were used instead of sample.

2.5. Statistical Analysis. All analyses were performed in sextuplicate (unless indicated otherwise) and all statistics were calculated using one way analysis of variance (ANOVA) on a JMP 12 statistical software (SAS Institute Inc., Cary, NC). Sample means were compared using Tukey HSD method and significant differences determined at $p \leq 0.05$. Correlations among parameters were computed using Pearson's correlation test.

\section{Results and Discussion}

The effects of WEAX on starch hydrolysis are presented in Figure 1. We compared the amount of glucose released over 30 minutes of incubation with $\alpha$-amylase in the presence or absence of WEAX fractions. Addition of WEAX fractions

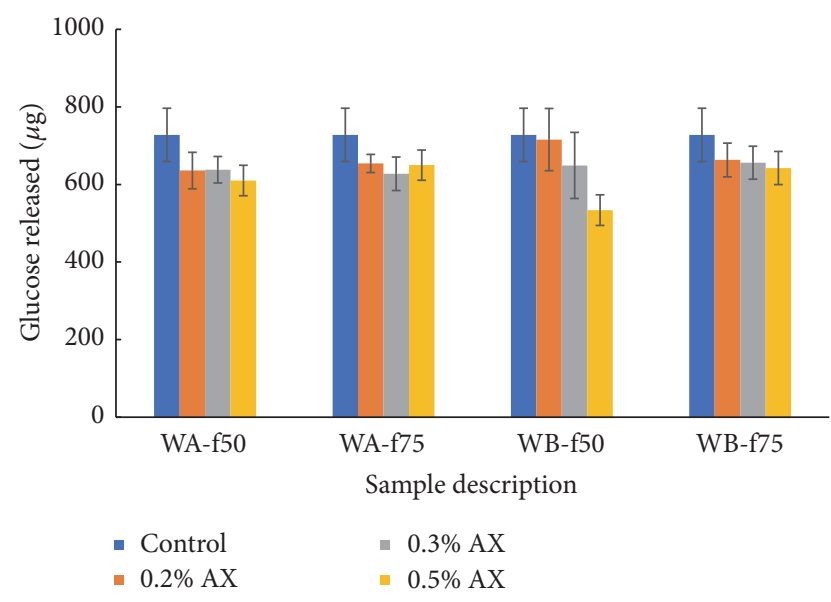

FIGURE 1: Effect of water extractable arabinoxylan on starch hydrolysis.

TABLE 2: Effect of feruloylated arabinoxylan on intestinal $\alpha$ glucosidase when sucrose or maltose was used as substrate.

\begin{tabular}{lcc}
\hline & \multicolumn{2}{c}{ IC50 $(\mathrm{mg} / \mathrm{mL})$} \\
\hline MAltose $_{50}$ & $4.88 \pm 0.30^{\mathrm{d}}(0.49)$ & Sucrose \\
WA-f $_{75}$ & $10.14 \pm 0.56^{\mathrm{a}}(1.01)$ & $*$ \\
WB-f $_{50}$ & $5.73 \pm 0.19^{\mathrm{c}}(0.57)$ & $*$ \\
WB-f $_{75}$ & $8.15 \pm 0.46^{\mathrm{b}}(0.81)$ & $*$ \\
Acarbose & $0.005 \pm 0.00^{\mathrm{e}}(\sim 0.0005)$ & $0.003 \pm 0.00$
\end{tabular}

Values presented as mean \pm standard deviation $(n=6)$. Data in the same column with the same superscript are not significantly different at $p \leq 0.05$. Data in parenthesis are IC50 values in \% w/v. IC50 value is the sample concentration resulting in $50 \%$ inhibition of $\alpha$-glucosidase activity. WA- $\mathrm{f}_{50}$ and WA- $\mathrm{f}_{75}$ : water-extractable fractions from WA obtained at 50 and $75 \%$ ammonium sulphate saturation, respectively. $W B-\mathrm{f}_{50}$ and $\mathrm{WB}-\mathrm{f}_{75}$ : waterextractable from WB obtained at 50 and $75 \%$ ammonium sulphate saturation, respectively.

numerically decreased the amount of glucose produced compared to control treatment. However, statistical comparisons of treatment groups and control showed that the mean difference was not significant $(p<0.05)$ for WA- $\mathrm{f}_{50}$, WA$\mathrm{f}_{75}$, and $\mathrm{WB}-\mathrm{f}_{75}$ regardless of WEAX concentration. The presence of $0.5 \% \mathrm{WB}^{-} \mathrm{f}_{50}$ resulted in significant decrease in amylolysis compared to control $(p<0.05)$. However, our observations for alpha amylase activity contrasted other reports in literature possibly due to concentration and type of AX. Amylolysis of starch was performed in the presence of 1 and $2 \% \mathrm{AX}$ and the AX used was devoid of ferulic acid [12]. We used concentrations of $\mathrm{AX}(\sim 5-10 \mathrm{~g})$ equivalent to that reported to attenuate postprandial blood glucose in human studies [1-3].

Table 2 shows the effect of WEAX on $\alpha$-glucosidase activity in the presence of sucrose or maltose as substrate. The data is presented as IC50 which is the concentration of the inhibitor resulting in $50 \%$ inhibition of $\alpha$-glucosidase activity. The IC50 values ranged from $4.88-10.14 \mathrm{mg} / \mathrm{mL}$ against $\alpha$-glucosidase activity with maltose as substrate. However, no inhibition was observed when sucrose was used as a substrate. 
TABLE 3: Correlation coefficient of arabinoxylans' inhibition of $\alpha$ glucosidase activity and its structural properties.

\begin{tabular}{lc}
\hline & $\alpha$-Glucosidase activity (IC50) \\
\hline Arabinose to xylose ratio & -0.67 \\
Ferulic acid content & -0.89 \\
Unsub-Xylp & 0.69 \\
Mono-Xyl $p$ at C (O)-32 & -0.86 \\
Mono-Xyl $p$ at C (O)-3 & -0.51 \\
Total Mono-Xylp & -0.76 \\
Di-Xylp & -0.63 \\
Molecular weight & 0.23 \\
Uronic acid & 0.36 \\
\hline
\end{tabular}

Data represent Pearson correlation coefficient values at $p \leq 0.05$. un-Xylp: unsubstituted xylose residues, mono-Xylp: monosubstituted xylose residue, di-Xylp: C (O)-2 and C (O)-3 disubstituted xylose residues.

The inhibitory potency of WEAX against intestinal maltase was 1000-2000 times less compared to acarbose (a positive control). It is widely hypothesised that viscosity may be the cause of the effect of AX on postprandial glucose [22]. Thus Vogel et al. fed rats a modified wheat bran AX to study the effect of viscosity [8 AXs were modified through oxidative gelation to increase their viscosity. Rats fed on modified AX had significantly lower postprandial blood glucose but not those fed on native AX demonstrating the role of viscosity. However, in this case, we used similar concentration of WEAX in both $\alpha$-amylase and $\alpha$-glucosidase activity studies and yet only the later was inhibited. This may imply that the effect of WEAX fractions on $\alpha$-glucosidase activity may not have been a consequence of viscosity but rather substrateenzyme-inhibitor interaction. The same is supported by the observation that sucrose activity of $\alpha$-glucosidase was not affected by the presence of WEAX in our study. Thus it is possible that the antiglycemic effect of AX may be due to inhibition of $\alpha$-glucosidase activity. Even though its potency on $\alpha$-glucosidase activity is 1000-2000 times less compared to acarbose, IC50 concentration is achievable upon consumption recommended dietary requirement of fiber (21-38 g/day).

The inhibition potency varied significantly $(p<0.05)$ among the WEAX samples with WA- $\mathrm{f}_{50}$ being the most potent. WEAX isolated at $50 \%$ ammonium sulphate saturation exhibited higher inhibition capacity compared to their corresponding fractions obtained at $75 \%$. A Pearson correlation analysis (Table 3) suggested that ferulic acid content, arabinose to xylose ratio, and pattern of xylose substitution may have influenced the inhibition activity of WEAX. Ferulic acid content of AX was a major determinant $(R=-0.89)$ of their inhibition potency. Antidiabetic properties of plant extracts have been associated with their total phenolic compound content $[25,26]$. Also, inhibition of intestinal alpha glucosidase by AX mono-/oligosaccharide was also related to their ferulic acid moiety [27]. Hence, WA$\mathrm{f}_{50}$ and $\mathrm{WB}-\mathrm{f}_{75}$ had significantly different inhibition potency towards $\alpha$-glucosidase activity despite having similar relative proportions unsubstituted (un-Xylp), monosubstituted,

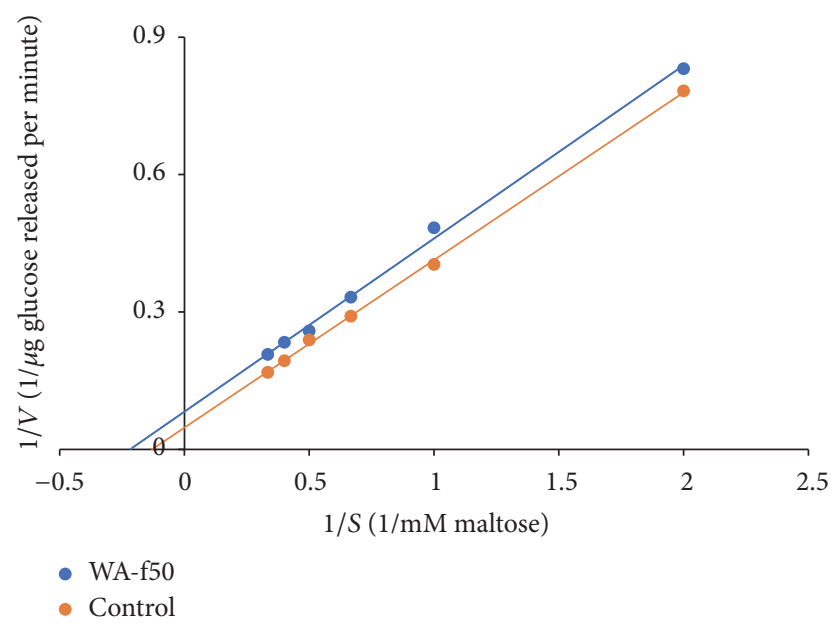

Figure 2: Lineweaver-Burk plot of rat intestinal $\alpha$-glucosidase inhibition by water extractable arabinoxylan $\left(\mathrm{WA}_{-\mathrm{f} 50}\right)$.

(2-Xylp or 3-Xylp), and disubstituted xylose residues (2,3$\mathrm{Xyl} p$ ) and degree of substitution but different ferulic acid content.

Arabinose to xylose ratio is a measure of degree of substitution (DS). A strong negative linear association ( $R$ $=-0.67)$ was observed between DS and IC50. This could have been a consequence of increased solubility of AX due to high DS. Thus highly substituted WEAX seems to have lower IC50 value (high inhibition potency). The same observation was supported by negative association between inhibition potency and relative proportion of unsubstituted xylose residues. The apparent di-Xyl ${ }_{\mathrm{p}}$ to $\mathrm{mono}_{\mathrm{Xyl}} \mathrm{\textrm {p }}$ residues ratio did not seem to have influence but the extent of xylose substitution showed an effect. Thus it is likely that the effect on $\alpha$-glucosidase activity may emanate from arabinose residues of WEAX. There have been reports on arabinose inhibiting $\alpha$-glucosidase activity [28]. Attempts to remove arabinose residues from WEAX using arabinofuranosidase were not successful in order to prove the hypothesis. However, we noted that mono- $\mathrm{Xyl}_{\mathrm{p}}$ at $\mathrm{C}(\mathrm{O})-2$ was a major determinant compared to mono- $\mathrm{Xyl}_{\mathrm{p}}$ at $\mathrm{C}(\mathrm{O})-3$ suggesting that inhibition potency was beyond the mere presence of arabinose residue. There was a strong correlation between mono- $\mathrm{Xyl}_{\mathrm{p}}$ at $\mathrm{C}(\mathrm{O})-2$ and ferulic acid content $(R=0.99)$. Thus it is possible that the observed influence of DS could have been derived from that of ferulic acid.

The Lineweaver-Burk plot (Figure 2) was used to calculate the apparent maximum velocity $\left(V_{\max }\right)$ and Michaelis-Menten constant $\left(K_{\mathrm{m}}\right)$ for $\alpha$-glucosidase activity on maltose in the presence and absence of WEAX. The effect of WEAX fraction on $V_{\max }$ and $K_{\mathrm{m}}$ was analysed to determine the type of inhibition. $V_{\max }$ and $K_{\mathrm{m}}$ of $\alpha$ glucosidase for maltose in absence of WEAX fractions were $17.5 \mu \mathrm{g}$ glucose per minute and $5.99 \mathrm{mM}$, respectively. Table 4 shows that addition of WEAX fractions decreased both $V_{\max }$ and $K_{\mathrm{m}}$ values suggesting that WEAX inhibited $\alpha$-glucosidase activity through an uncompetitive mode. A typical characteristic of uncompetitive inhibition is that both 
TABLE 4: Inhibition kinetics of water extractable arabinoxylans derived from Lineweaver-Burk plots.

\begin{tabular}{lcc}
\hline & $V_{\max }(\mu$ glucose $/$ minute $)$ & $K_{\mathrm{m}}(\mathrm{mM}$ maltose $)$ \\
\hline Control & $17.5 \pm 0.48^{\mathrm{a}}$ & $5.99 \pm 0.16^{\mathrm{a}}$ \\
WA-f & $12.07 \pm 0.22^{\mathrm{c}}$ & $4.54 \pm 0.08^{\mathrm{c}}$ \\
WA-f $_{75}$ & $16.73 \pm 0.42^{\mathrm{a}}$ & $5.07 \pm 0.13^{\mathrm{b}}$ \\
WB-f $_{50}$ & $\mathrm{Nd}$ & $\mathrm{nd}$ \\
WB- $_{75}$ & $14.73 \pm 0.33^{\mathrm{b}}$ & $4.91 \pm 0.11^{\mathrm{b}}$ \\
\hline
\end{tabular}

Values presented as mean \pm standard deviation $(n=3)$. Data in the same column with the same superscript are not significantly different at $p \leq$ $0.05 . V_{\max }=$ maximum velocity; $K_{\mathrm{m}}$ is the Michalelis-Menten constant (substrate concentration required for an enzyme to reach half $V_{\max }$ ). nd means not determined. WA- $\mathrm{f}_{50}$ and $\mathrm{WA}-\mathrm{f}_{75}$ : water-extractable fractions from WA obtained at 50 and $75 \%$ ammonium sulphate saturation, respectively. $\mathrm{WB}-\mathrm{f}_{50}$ and $\mathrm{WB}-\mathrm{f}_{75}$ : water-extractable from WB obtained at 50 and $75 \%$ ammonium sulphate saturation, respectively.

$V_{\max }$ and $K_{\mathrm{m}}$ decrease in the presence of inhibitor. Thus it is plausible that WEAX fractions bind to enzyme substrate complex thereby decreasing both $V_{\max }$ and $K_{\mathrm{m}}$. Arabinose was also found to inhibit $\alpha$-glucosidase activity through an uncompetitive mode [28].

Our results may provide an explanation on the inconsistency observed in literature about the effect of AX on postprandial glucose level. Feeding of Zuker diabetic rats with AX (Ara/Xyl = 0.9) supplemented bread resulted in a significant decrease in postprandial blood glucose level [7]. In contrast, intake of native $\mathrm{AX}(\mathrm{Ara} / \mathrm{Xyl}=0.5)$ had no effect on blood glucose response [8]. Also, supplementation of diets with 6 and $12 \mathrm{~g} \mathrm{AX}$ (Ara/Xyl $=0.66$ or 0.8 ) decreased blood glucose in both healthy and diabetic subjects $[1,2,5]$. However, AX (Ara/Xyl = 0.8) did not attenuate postprandial glucose response in healthy human adults [6]. Pigs fed on white bread supplemented with AXs had a reduced net glucose flux compared to pigs fed on white bread [29]. The absence of detailed chemical composition and structures makes it difficult to compare the results on effectiveness of AX [22]. Thus even though concentrations of AXs used may be the same, its effectiveness would depend on the nature of AXs used. We have demonstrated that AX obtained at 50\% ammonium sulphate saturation exhibited higher inhibition potency compared to AX obtained at $75 \%$.

\section{Conclusion}

The results of this study indicated that antiglycemic effect of arabinoxylans may be derived from inhibiting intestinal $\alpha$-glucosidase activity but not amylase activity. The potency of water extractable AX on $\alpha$-glucosidase activity was influenced by ferulic acid contents arabinose to xylose ratio and pattern of xylose substitution. The findings also suggest that inhibition of $\alpha$-glucosidase activity occurs through uncompetitive mechanism. Thus, consumption of diet rich in water extractable AX may attenuate postprandial blood glucose level.

\section{Disclosure}

Part of the results was presented as an oral poster at the Functional Foods and Natural Health Products Graduate Research (FFNHP) symposium/Therapeutic Applications of Functional Foods and Bioactives (TAFFB) conference held at the St-Boniface Hospital Albrechtsen Research Centre from April 20 to April 22, 2016.

\section{Conflicts of Interest}

All funds or material support received did not lead to conflicts of interest in publication of this manuscript.

\section{Acknowledgments}

This research was funded by the Natural Sciences and Engineering Research Council of Canada (NSERC) through the Discovery Grant Programme. The authors are also grateful to Cargill Limited for provision of wheat aleurone samples and Ce Zhou, Alison Ser, and Pat Kenyon of the Department of Food Science, University of Manitoba, for their technical support.

\section{References}

[1] Z. X. Lu, K. Z. Walker, J. G. Muir, T. Mascara, and K. O’Dea, "Arabinoxylan fiber, a byproduct of wheat flour processing, reduces the postprandial glucose response in normoglycemic subjects," The American Journal of Clinical Nutrition, vol. 71, no. 5, pp. 1123-1128, 2000.

[2] A. L. Garcia, B. Otto, S.-C. Reich et al., "Arabinoxylan consumption decreases postprandial serum glucose, serum insulin and plasma total ghrelin response in subjects with impaired glucose tolerance," European Journal of Clinical Nutrition, vol. 61, no. 3, pp. 334-341, 2007.

[3] A. L. Garcia, J. Steiniger, S. C. Reich et al., "Arabinoxylan fibre consumption improved glucose metabolism, but did not affect serum adipokines in subjects with impaired glucose tolerance," Hormone and Metabolic Research, vol. 38, no. 11, pp. 761-766, 2006.

[4] H. Hanai, M. Ikuma, Y. Sato et al., "Long-term effects of watersoluble corn bran hemicellulose on glucose tolerance in obese and non-obese patients: improved insulin sensitivity and glucose metabolism in obese subjects," Bioscience, Biotechnology, and Biochemistry, vol. 61, no. 8, pp. 1358-1361, 1997.

[5] Z. X. Lu, K. Z. Walker, J. G. Muir, and K. O’Dea, “Arabinoxylan fibre improves metabolic control in people with type II diabetes," European Journal of Clinical Nutrition, vol. 58, no. 4, pp. 621-628, 2004.

[6] M. Möhlig, C. Koebnick, M. O. Weickert et al., "Arabinoxylanenriched meal increases serum ghrelin levels in healthy humans," Hormone and Metabolic Research, vol. 37, no. 5, pp. 303-308, 2005.

[7] M. L. Hartvigsen, P. B. Jeppesen, H. N. Lærke, E. N. Njabe, K. E. B. Knudsen, and K. Hermansen, "Concentrated arabinoxylan in wheat bread has beneficial effects as rye breads on glucose and changes in gene expressions in insulin-sensitive tissues of Zucker diabetic fatty (ZDF) rats," Journal of Agricultural and Food Chemistry, vol. 61, no. 21, pp. 5054-5063, 2013.

[8] B. Vogel, D. D. Gallaher, and M. Bunzel, "Influence of cross-linked arabinoxylans on the postprandial blood glucose 
response in rats," Journal of Agricultural and Food Chemistry, vol. 60, no. 15, pp. 3847-3852, 2012.

[9] D. J. A. Jenkins, T. M. S. Wolever, A. R. Leeds et al., "Dietary fibres, fibre analogues, and glucose tolerance: importance of viscosity," British Medical Journal, vol. 1, no. 6124, pp. 1392-1394, 1978.

[10] M. S. Izydorczyk and C. G. Biliaderis, "Cereal arabinoxylans: advances in structure and physicochemical properties," Carbohydrate Polymers, vol. 28, no. 1, pp. 33-48, 1995.

[11] M. S. Kale, M. P. Yadav, K. B. Hicks, and K. Hanah, "Concentration and shear rate dependence of solution viscosity for arabinoxylans from different sources," Food Hydrocolloids, vol. 47, pp. 178-183, 2015.

[12] S. Dhital, G. Dolan, J. R. Stokes, and M. J. Gidley, "Enzymatic hydrolysis of starch in the presence of cereal soluble fibre polysaccharides," Food \& Function, vol. 5, no. 3, pp. 579-586, 2014.

[13] M.-G. Ma, N. Jia, J.-F. Zhu, S.-M. Li, F. Peng, and R.-C. Sun, "Isolation and characterization of hemicelluloses extracted by hydrothermal pretreatment," Bioresource Technology, vol. 114, pp. 677-683, 2012.

[14] M. M. Smith and R. D. Hartley, "Occurrence and nature of ferulic acid substitution of cell-wall polysaccharides in graminaceous plants," Carbohydrate Research, vol. 118, pp. 65-80, 1983.

[15] M. Bunzel, J. Ralph, and H. Steinhart, "Association of nonstarch polysaccharides and ferulic acid in grain amaranth (Amaranthus caudatus L.) dietary fiber," Molecular Nutrition and Food Research, vol. 49, no. 6, pp. 551-559, 2005.

[16] C. Antoine, S. Peyron, F. Mabille et al., "Individual contribution of grain outer layers and their cell wall structure to the mechanical properties of wheat bran," Journal of Agricultural and Food Chemistry, vol. 51, no. 7, pp. 2026-2033, 2003.

[17] K. Gebruers, E. Dornez, D. Boros et al., "Variation in the content of dietary fiber and components thereof in wheats in the healthgrain diversity screen," Journal of Agricultural and Food Chemistry, vol. 56, no. 21, pp. 9740-9749, 2008.

[18] A. C. Dona, G. Pages, R. G. Gilbert, and P. W. Kuchel, "Digestion of starch: in vivo and in vitro kinetic models used to characterise oligosaccharide or glucose release," Carbohydrate Polymers, vol. 80, no. 3, pp. 599-617, 2010.

[19] L. Sim, C. Willemsma, S. Mohan, H. Y. Naim, B. M. Pinto, and D. R. Rose, "Structural basis for substrate selectivity in human maltase-glucoamylase and sucrase-isomaltase N-terminal domains," The Journal of Biological Chemistry, vol. 285, no. 23, pp. 17763-17770, 2010.

[20] E. M. Wright, B. A. Hirayama, and D. F. Loo, "Active sugar transport in health and disease," Journal of Internal Medicine, vol. 261, no. 1, pp. 32-43, 2007.

[21] C. F. Burant, J. Takeda, E. Brot-Laroche, G. Bell, and N. Davidson, "Fructose transporter in human spermatozoa and small intestine is GLUT5," Journal of Biological Chemistry, vol. 267, no. 21, pp. 14523-14526, 1992.

[22] R. Gemen, J. F. De Vries, and J. L. Slavin, "Relationship between molecular structure of cereal dietary fiber and health effects: focus on glucose/insulin response and gut health," Nutrition Reviews, vol. 69, no. 1, pp. 22-33, 2011.

[23] M. S. Izydorczyk and C. G. Biliaderis, "Studies on the structure of wheat-endosperm arabinoxylans," Carbohydrate Polymers, vol. 24, no. 1, pp. 61-71, 1994.

[24] T. Oki, T. Matsui, and Y. Osajima, "Inhibitory effect of $\alpha$ glucosidase inhibitors varies according to its origin," Journal of
Agricultural and Food Chemistry, vol. 47, no. 2, pp. 550-553, 1999.

[25] H. Zhang, G. Wang, T. Beta, and J. Dong, "Inhibitory properties of aqueous ethanol extracts of propolis on alpha-glucosidase," Evidence-Based Complementary and Alternative Medicine, vol. 2015, Article ID 587383, 7 pages, 2015.

[26] K. Tadera, Y. Minami, K. Takamatsu, and T. Matsuoka, "Inhibition of $\alpha$-glucosidase and $\alpha$-amylase by flavonoids," Journal of Nutritional Science and Vitaminology, vol. 52, no. 2, pp. 149-153, 2006.

[27] L. N. Malunga, P. Eck, and T. Beta, "Inhibition of intestinal $\alpha$ glucosidase and glucose absorption by feruloylated arabinoxylan mono- and oligosaccharides from corn bran and wheat aleurone," Journal of Nutrition and Metabolism, vol. 2016, Article ID 1932532, 9 pages, 2016.

[28] K. Seri, K. Sanai, N. Matsuo, K. Kawakubo, C. Xue, and S. Inoue, "L-arabinose selectively inhibits intestinal sucrase in an uncompetitive manner and suppresses glycemic response after sucrose ingestion in animals," Metabolism: Clinical and Experimental, vol. 45, no. 11, pp. 1368-1374, 1996.

[29] K. L. Christensen, M. S. Hedemann, H. N. Lærke et al., "Concentrated arabinoxylan but not concentrated $\beta$-glucan in wheat bread has similar effects on postprandial insulin as whole-grain rye in porto-arterial catheterized pigs," Journal of Agricultural and Food Chemistry, vol. 61, no. 32, pp. 7760-7768, 2013. 


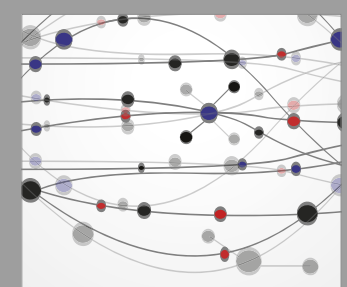

The Scientific World Journal
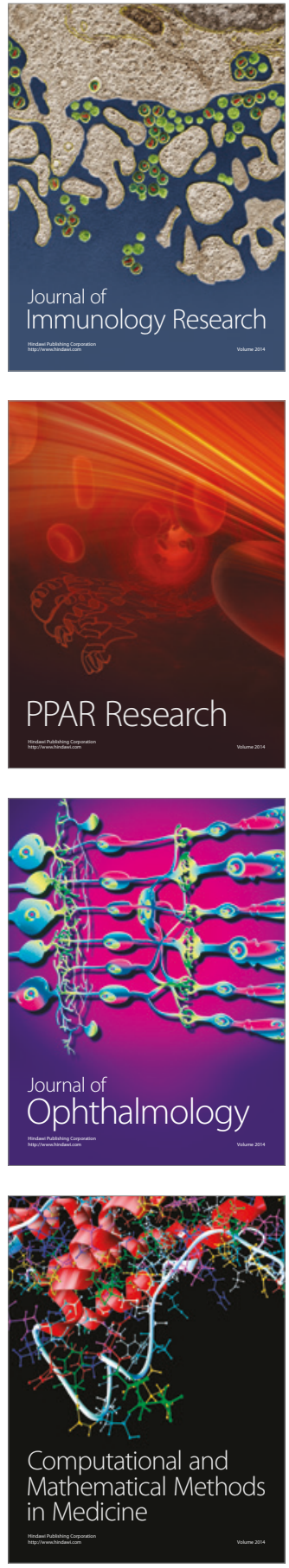

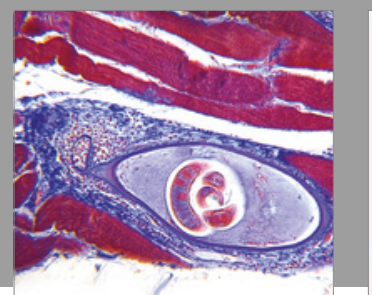

Gastroenterology Research and Practice
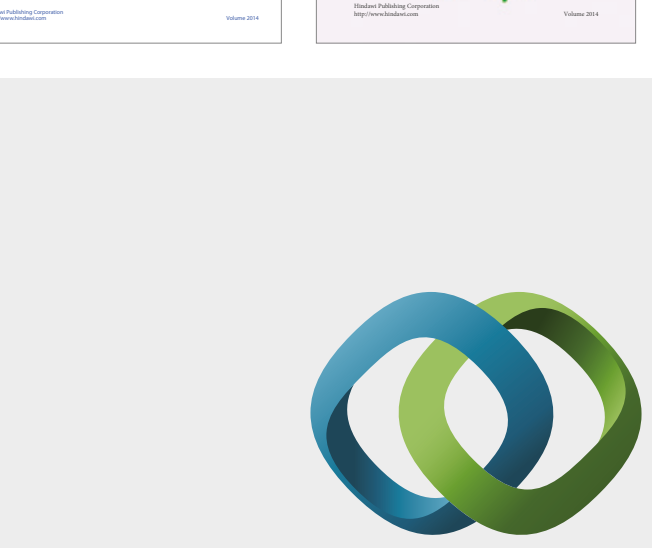

\section{Hindawi}

Submit your manuscripts at

https://www.hindawi.com
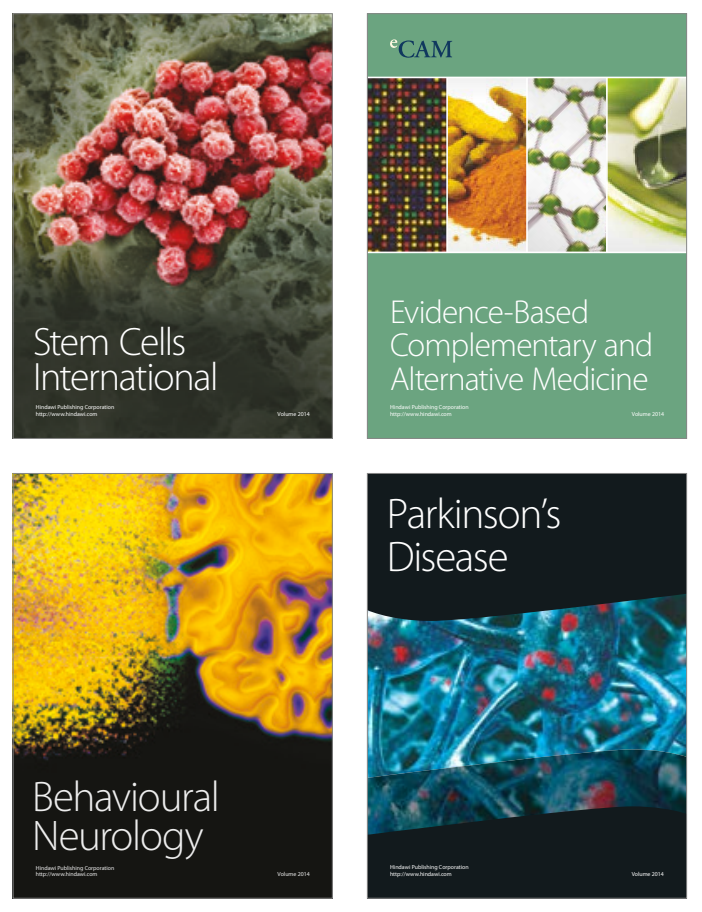
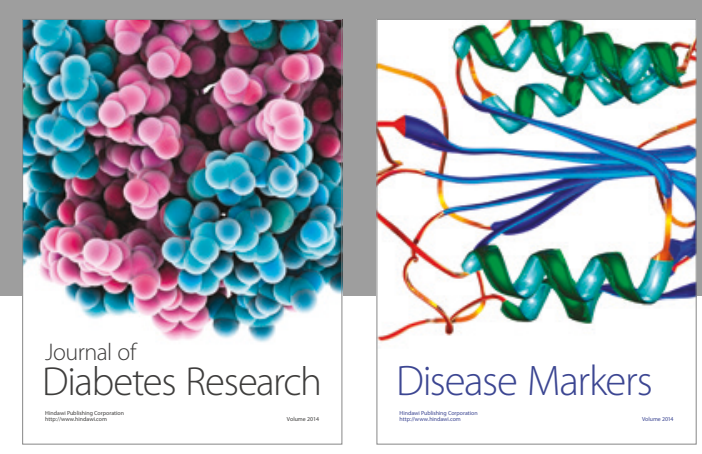

Disease Markers
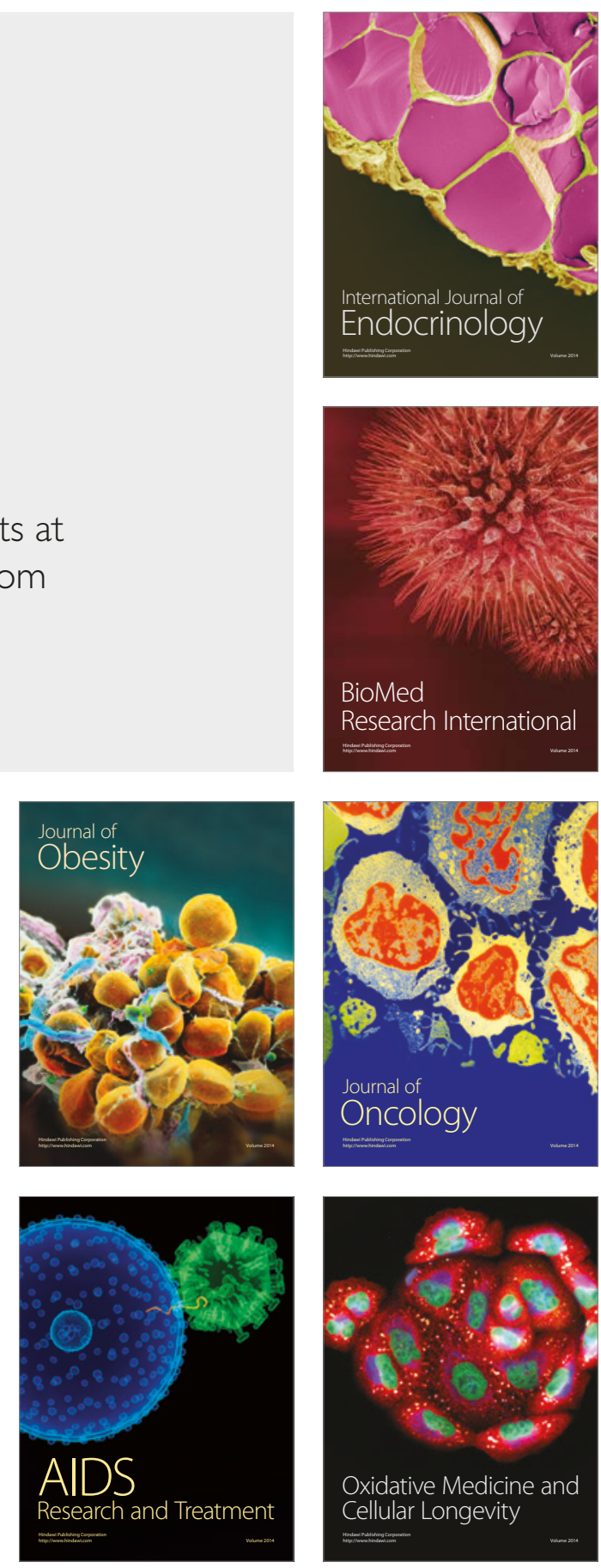